\title{
Efimov and Thomas effects and the model dependence of three-particle observables in two and three dimensions
}

\author{
Sadhan K. Adhikari \\ Departamento de Física, Universidade Federal de Pernambuco, 50.739 Recife, Pernambuco, Brazil
}

A. Delfino

Instituto de Física, Universidade Federal Fluminense, 24.210 Niterói, Rio de Janeiro, Brazil

T. Frederico

Centro Técnico Aeroespacial, Instituto de Estudos Avançados, 12.200 São José dos Campos, São Paulo, Brazil

I. D. Goldman

Instituto de Física, Universidade de São Paulo, 01.498 São Paulo, São Paulo, Brazil

Lauro Tomio

Instituto de Física Teórica, Universidade Estadual Paulista, Rua Pamplona 145, 01.405 São Paulo, São Paulo, Brazil

(Received 28 August 1987)

\begin{abstract}
It is demonstrated for the three-dimensional three-particle system that a divergence arising from essentially the same singularity structure of the kernel of the scattering integral equation is responsible for both the Efimov and Thomas effects. The above divergence implies that the results of a three-particle dynamical calculation will be sensitive to the details of the two-particle interaction. In two-dimensional systems, the above divergence is absent and consequently the three-particle observables become essentially independent of the two-particle interaction model.
\end{abstract}

\section{INTRODUCTION}

Over the past decade there has been considerable interest in two-dimensional systems such as helium adsorbed on graphite ${ }^{1}$ and spin-polarized hydrogen $(\mathbf{H} \downarrow)$ recombining on a helium film. ${ }^{2}$ The theoretical treatment such as systems involves two-dimensional quantum-mechanical problems sometimes involving three or more particles. In view of the possibility of detecting multiparticle bound states in monolayers of quantum gases, it is interesting to solve numerically the boundstate problem for a few particles in two dimensions. ${ }^{3}$ Such numerical calculations have already been done by Tjon $^{3}$ and others (see Ref. 4). Also, a study of twodimensional quantum-mechanical three-particle systems is essential ${ }^{5}$ for studying low-temperature behavior of quantum cluster coefficients in two dimensions, which is crucial for studying the quantum-mechanical properties of such systems. In order to have a complete understanding of the above problems, it is necessary to study the quantum-mechanical three-particle problem both formally and numerically.

From the experience gained from the study of threedimensional three-particle quantum systems, we shall demonstrate certain general features of the quantummechanical three-particle system in two dimensions. In particular, we shall show that, if reasonable assumptions are made about the underlying two-particle interaction, the quantum-mechanical three-particle problem in two dimensions is basically independent of the two-particle interaction model. We shall also demonstrate that this model independence is a consequence of the absence of the Efimov ${ }^{6}$ and the Thomas ${ }^{7}$ effects in two dimensions. The three-particle three-dimensional problem, on the other hand, is very sensitive to the model employed in its solution. In this paper we present a unified treatment of Efimov and Thomas effects and the model dependence of three-particle observables in two and three dimensions.

The quantum-mechanical three-dimensional threeparticle system is the simplest one which shows certain peculiar effects and has become the subject of intense study in atomic, nuclear, and particle physics especially because such a study is expected to yield information about the underlying two-particle interaction. The two most remarkable properties that this system exhibits were first studied by Efimov and Thomas and are known as the Efimov $^{6}$ and the Thomas ${ }^{7}$ effects, respectively.

Efimov $^{6}$ showed that if three nonrelativistic identical bosons interact via a short-range two-particle interaction $\lambda V(r)$ characterized by a range parameter $r_{0}$, then in the limit as $\lambda \rightarrow \lambda_{0}$ where $\lambda_{0}$ is the strength needed to support the first zero-energy two-particle bound state, the number of three-particle $s$-wave bound states $N$ tends to infinity and is roughly given by

$$
N \sim \pi^{-1} \ln \left(|a| / r_{0}\right),
$$

where $a$ is the two-particle scattering length which tends to infinity in this limit. (There are two equivalent ways of reaching this limit $\lambda \rightarrow \lambda_{0}$, one from above $\left(\lambda>\lambda_{0}\right.$, $a \rightarrow+\infty)$ and one from below $\left(\lambda<\lambda_{0}, a \rightarrow-\infty\right)$. How ever, for our mathematical treatment of the problem 
these two limits are equivalent and lead to the same divergence of the trace of the kernel of the Faddeev equation [see discussion after Eq. (7)].) In a different context Thomas ${ }^{7}$ showed that if three nonrelativistic particles interact via short-range two-particle interaction $\lambda V(r)$, then as the range $r_{0}$ of this interaction tends to zero, the binding energy of the three-particle system $B$ increases beyond any limit: $B \rightarrow \infty$. We shall show in this work that these two apparently different effects are related essentially to the same divergence of the trace of the kernel of the scattering equation of the three-particle system in two different limits and, hence, have the same mathematical origin. We demonstrate from our unified discussion of the Efimov and Thomas effects that as $r_{0} \rightarrow 0$ in the Thomas limit, the three-particle system is expected to have infinite number of bound states with an accumulation point at infinite binding energy. It is worthwhile to recall that as $|a| \rightarrow \infty$ in the Efimov limit, the infinite number of bound states accumulate at zero binding energy.

In our unified treatment of this problem, we show that the presence of one of the Efimov and Thomas effects implies the presence of the other. Also, we expect that the study of the three-particle system in three dimensions at low energies, but slightly away from the Efimov limit, will yield information about the underlying two-particle interaction, and especially about its off-shell behavior. This is related to the existence of the above-mentioned divergence of the trace of the kernel responsible for the Efimov and Thomas effects. In other words, this divergence of the trace of the kernel implies that the threeparticle observables be model dependent (i.e., sensitive to the off-shell behavior of two-particle interaction) when one is slightly away from the Efimov limit.

We verify that in two-dimensional three-particle systems the Efimov-Thomas divergence of the trace of the kernel is absent and consequently there are no Efimov or Thomas effects, and for this system the low-energy threeparticle observables are less sensitive to the off-shell behavior of two-particle interaction and are reasonably model independent. The low-energy four-particle system (and most likely the $n$-particle system) in two dimensions is also expected to yield model-independent results if onshell two-particle properties are held fixed. The threedimensional low-energy four-particle system is, on the other hand, sensitive to the off-shell behavior of twoparticle interaction.

The present discussion has an interesting consequence on the low-energy three-nucleon system. In this case the kernel of the underlying scattering equation shows the Efimov-Thomas divergence and the expected off-shell sensitivity of low-energy three-nucleon observables has been observed in numerical calculations. ${ }^{8}$ It has been shown $^{6,9}$ that in the Efimov limit the three-particle system in three dimensions can essentially be described in terms of an effective $r^{-2}$ type interaction at large distances. Moreover, the properties of the infinite number of Efimov states become independent of the details of the two-particle interaction. As we move away from the Efimov limit (by increasing the deuteron binding for instance) the remaining three-particle bound states are ex- pected to become more and more model dependent. In the case of the three-nucleon system, as the deuteron binding is "low" and as one is still near the Efimov limit, the off-shell sensitivity of low-energy three-nucleon observables is not very strong and essentially is controlled by one three-nucleon parameter ${ }^{6-9}$ such as the triton binding energy. Once two on-shell equivalent twonucleon interactions produce identical triton binding, they produce identical results for all three-nucleon observables. This leads to correlations among various theoretically calculated trinucleon observables, ${ }^{8,10}$ which have been observed in various realistic and model calculations.

In two dimensions we expect the three-particle observables to be reasonably insensitive to off-shell properties of the two-particle interaction. This model independence of the three-particle system in two dimensions was implicit in the work of Tjon ${ }^{3}$ who, however, was not completely aware of it. We shall explicitly demonstrate this model independence numerically and provide a physical explanation for it through our unified discussion of the Efimov and Thomas effects.

The plan of the paper is as follows. In Sec. II we present our unified description of the Efimov and Thomas effects in three dimensions and its relation with the offshell sensitivity of three-particle observables. In Sec. III we present a similar discussion in two dimensions and provide justification of model independence of threeparticle observables in two dimensions. In Sec. IV we present some numerical results for the three-particle energy in two dimensions and demonstrate its model independence. Finally, in Sec. V we present a brief discussion and concluding remarks.

\section{THE EFIMOV AND THOMAS EFFECTS IN THREE DIMENSIONS}

For simplicity we limit our discussions in this section and the following to the case of three identical bosons. As we shall base our discussion on the work of Amado and Noble, ${ }^{11}$ we present only a brief account of their work in the following and refer the more interested reader to the original article.

The homogeneous three-particle scattering equations responsible for the bound-state problem reduce to a single equation in the case of identical bosons each of mass $m$, which can be written as

$$
\Psi(\mathbf{p}, \mathbf{k})=\int d^{3} p^{\prime} d^{3} k^{\prime} K\left(\mathbf{p}, \mathbf{k} ; \mathbf{p}^{\prime}, \mathbf{k}^{\prime}\right) \bar{\Psi}\left(\mathbf{p}^{\prime}, \mathbf{k}^{\prime}\right),
$$

where in units $\hbar=m=1$, the kernel $K$ is given by

$$
\begin{aligned}
K\left(\mathbf{p}, \mathbf{k} ; \mathbf{p}^{\prime}, \mathbf{k}^{\prime}\right)= & \frac{2\left\langle\mathbf{k}+\frac{1}{2} \mathbf{p}\left|t\left(E-\frac{3}{4} p^{2}\right)\right| \mathbf{p}^{\prime}+\frac{1}{2} \mathbf{k}^{\prime}\right\rangle}{E-p^{2}-k^{2}-\mathbf{p} \cdot \mathbf{k}} \\
& \times \delta^{3}\left(\mathbf{p}-\mathbf{k}^{\prime}\right),
\end{aligned}
$$

where $t$ is the two-particle $t$ matrix and $E$ is the threeparticle center-of-mass energy. It has been suggested that since the divergence of the trace of the kernel at the Efimov limit arises from the small momentum behavior of the kernel, the essential features of the Efimov effect 
can be obtained by keeping only the pole term of the two-particle $t$ matrix. Equation (2) then reduces to a one-variable integral equation

$$
\Psi(\mathbf{p})=\int d^{3} p^{\prime} \hat{K}\left(\mathbf{p}, \mathbf{p}^{\prime} ; E, \lambda\right) \Psi\left(\mathbf{p}^{\prime}\right),
$$

with

$$
\begin{aligned}
\hat{K}\left(\mathbf{p}, \mathbf{p}^{\prime} ; E, \lambda\right)=- & \pi^{2} \Theta(\Lambda-p) \Theta\left(\Lambda-p^{\prime}\right) \\
& \times\left[a^{-1}+\left(\frac{3}{4} p^{2}-E\right)^{1 / 2}\right]^{-1} \\
& \times\left(E-p^{2}-p^{\prime 2}-\mathbf{p} \cdot \mathbf{p}^{\prime}\right)^{-1},
\end{aligned}
$$

where $a$ is the usual two-particle scattering length and $\Lambda$ is a momentum cutoff introduced to preserve convergence at large momentum. $\Lambda$ is supposed to be related to the range $r_{0}$ of two-particle interaction by $\Lambda=r_{0}^{-1}$. The pole approximation for the off-shell two-particle $t$ matrix in Eq. (5) is consistent with the zero range limit, $r_{0} \rightarrow 0$. However, in order to reach the Thomas limit the inverse range parameter of Eq. (5) should tend to infinity consistent with $r_{0}=\Lambda^{-1}=0$. The Efimov limit is achieved by taking $|a| \rightarrow \infty$ in Eq. (5). Hence, by taking appropriate limits in Eqs. (4) and (5), one can study the Efimov and Thomas effects in a unified way.

For our discussion it is convenient to study the auxiliary eigenvalue problem

$$
\eta_{n}(E, \lambda) \phi_{n}(\mathbf{p} ; E, \lambda)=\int d^{3} p^{\prime} \widehat{K}\left(\mathbf{p}, \mathbf{p}^{\prime} ; E, \lambda\right) \phi_{n}\left(\mathbf{p}^{\prime} ; E, \lambda\right)
$$

For each $E$ and $\lambda$ there is an infinite set of discrete eigenvalues $\eta_{n}$ of Eq. (6). The consideration of the auxiliary eigenvalue problem (6) relates the divergence of $\operatorname{Tr} \widehat{K}^{n}$ with the appearance of the Efimov or the Thomas effect. For any $E$ and $\lambda, \eta_{n}=1$ corresponds to an eigenstate of the problem. For a fixed negative $E$ the real $\eta$ 's grow in magnitude with $\lambda$. Hence, each $\eta$ for a fixed $\lambda\left(<\lambda_{0}\right)$ can be unity once as $E$ goes from $-\infty$ to 0 . Thus an infinite number of bound states each with binding energy greater than $B$ must correspond to an infinite number of eigenvalues $\eta$ 's being greater than unity at $E=-B$, which will imply that $\operatorname{Tr} \widehat{K}^{n}(E=-B, \lambda)$ diverge for any $n$.

As the divergence of the trace of the kernel is a necessary condition for an infinite number of $s$-wave bound states to appear, we examine the trace of the $s$-wave projection of the kernel. The trace is given by

$\operatorname{Tr} \hat{K} \sim \int_{0}^{\Lambda} d p \ln \left(\frac{\beta^{2}+3 p^{2}}{\beta^{2}+p^{2}}\right)\left[\alpha+\left(\frac{3}{4} p^{2}+\beta^{2}\right)^{1 / 2}\right]^{-1}$,

where $\beta^{2}=-E$ and $\alpha=1 / a$. The divergent part of the integral (7) is independent of the sign of $\alpha$, and hence of $a$, and by using a dimensional argument it is easy to see from Eq. (7) that for $\beta^{2}=-E=0, \operatorname{Tr} \hat{K}$ diverges at the lower limit $(p \rightarrow 0)$ as $\ln |\alpha|$ when $|\alpha| \rightarrow 0$, and diverges at the upper limit $(p \rightarrow \Lambda)$ as $\ln \Lambda$ when $\Lambda \rightarrow \infty$. The first divergence corresponds to the Efimov limit and the second corresponds to the Thomas limit. It is easy to see that these two divergences have essentially the same mathematical origin and that one implies the other. For our purposes, we rewrite $\operatorname{Tr} \widehat{K}$ given by Eq. (7) as

$$
\begin{aligned}
\operatorname{Tr} \hat{K} \sim \int_{0}^{\Lambda / \alpha} d y \ln \left(\frac{\beta^{2} / \alpha^{2}+3 y^{2}}{\beta^{2} / \alpha^{2}+y^{2}}\right) \\
\times\left[1+\left(\frac{3}{4} y^{2}+\beta^{2} / \alpha^{2}\right)^{1 / 2}\right]^{-1}
\end{aligned}
$$

Now $\operatorname{Tr} \hat{K}$ of Eq. (8) is perfectly finite at the lower limit $y \rightarrow 0$, but may diverge at the upper limit as $\Lambda / \alpha \rightarrow \infty$. Hence, both the Efimov divergence and the Thomas divergence appear in the large $y$ divergence of Eq. (8). The essential divergence properties of $\operatorname{Tr} \hat{K}$ of Eq. (8) can be summarized as follows. For $\beta=0, \operatorname{Tr} \hat{K}$ diverges as $\ln (\Lambda / \alpha)$ when either $\alpha \rightarrow 0$ or $\Lambda \rightarrow \infty$; for $\beta \neq 0, \operatorname{Tr} \hat{K}$ diverges as $\ln \Lambda$ as $\Lambda \rightarrow \infty$ and remains finite as $\alpha \rightarrow 0$. In the above limiting procedure we, however, do not consider the possibility that $\alpha \rightarrow 0$ and $\Lambda \rightarrow \infty$ simultaneously. The divergence of $\operatorname{Tr} \hat{K}$ at $E=-B$ is only a necessary condition for the appearance of an infinite number of bound states with binding energy greater than $B$. In order that an infinite number of bound states really appears, one requires that $\operatorname{Tr} \hat{K}^{n}$ diverges as $\ln (\Lambda / \alpha)$ for all $n$ such that an infinite number of eigenvalues of $\widehat{K}$ accumulate at a value greater than unity. The necessary rigorous study of $\operatorname{Tr} \hat{K}{ }^{n}$ has been performed by Amado and Noble, ${ }^{11}$ and we do not repeat it here. From the above divergence of $\operatorname{Tr} \hat{K}$ for $\beta=0$, we conclude that there is an infinite number of bound states both in the Efimov limit $(\alpha \rightarrow 0)$ and in the Thomas limit $(\Lambda \rightarrow \infty)$. For any finite $\beta(\neq 0)$, $\operatorname{Tr} \widehat{K}$ does not diverge as $\alpha \rightarrow 0$, which means that in the Efimov limit there is not an infinite number of bound states with finite binding energy; and an infinite number of Efimov states accumulates at zero energy. But for large but finite $\beta=\beta_{0}, \operatorname{Tr} \hat{K}$ diverges as $\ln \Lambda$ as $\Lambda \rightarrow \infty$, which means that in the Thomas limit there is an infinite number of bound states with binding energy greater than $\beta_{0}^{2}$. As this is true for any $\beta_{0}$, however large, we conclude that the infinite number of Thomas states accumulate at infinite binding energy.

Apart from this similarity in the mathematical description of Efimov and Thomas effects, there is a physical similarity between these two effects. In the case of Efimov effect, $|a| \rightarrow \infty$ and $r_{0}$ is finite, whereas in the case of the Thomas effect, $r_{0} \rightarrow 0$ and $|a|$ is finite. In both cases $|a| \gg r_{0}$, a limit which can be achieved by making $|a| \rightarrow \infty$ (Efimov effect) or $r_{0} \rightarrow 0$ (Thomas effect). At $\beta=0 \operatorname{Tr} \widehat{K}$ diverges as $\ln \left(|a| / r_{0}\right)$. We call this divergence the Efimov-Thomas divergence, which happens for $|a| / r_{0} \rightarrow \infty$ in our unified discussion of the Efimov and Thomas effects. Both these effects can be easily understood in terms of the effective interaction of the system. It has been demonstrated in a simple model by Fonseca, Redish, and Shanley ${ }^{12}$ that the effective interaction of the system has a $1 / r^{2}$ behavior at intermediate distances for which $|a|>r>r_{0}$, where $r_{0}$ is the range of two-particle interaction and $a$ is the two-particle scattering length. When $|a| \rightarrow \infty$, we have a long-range effective $1 / r^{2}$ interaction which leads to the Efimov effect; and when $r_{0} \rightarrow 0$ we have a $1 / r^{2}$ interaction at short distance which leads to the collapse of the Thomas states at infinite binding. Hence both these effects can be 
understood by two limiting cases of the same effective interaction.

In a less pathological and more realistic situation at low energies for a finite $|a| \gg r_{0}(\neq 0)$, Eqs. (4) and (5) are still valid, but now with the $\Theta$ 's replaced by more realistic form factors. In this case the convergence of the integral in Eq. (4) is not given by the cutoff $\Lambda$, which is now set equal to infinity, but is given by the large momentum behavior of the form factors of the two-particle interaction. Then the solution of Eq. (4) is expected to be very sensitive to the large momentum behavior of the two-body interaction or to its short-range behavior. Essentially, once the two-particle on-shell properties are held fixed and the two-particle interaction is assumed to be of short range, this flexibility of the two-body interaction at short distances leads to different off-shell behavior, which in turn will lead to different three-particle observables. As mentioned in Sec. I this off-shell sensitivity of three-particle observables has been observed in threenucleon calculations. ${ }^{8-10}$ In general different on-shell equivalent two-nucleon interactions lead to distinct three-nucleon observables. ${ }^{8}$ The situation is completely different in two dimensions and we present a discussion of this case in the following section.

\section{THE ABSENCE OF THE EFIMOV AND THOMAS EFFECTS IN TWO DIMENSIONS}

In this section we shall deal with the absence of the Efimov and Thomas effects in two dimensions. In this case the $s$-wave projection of Eqs. (4) and (5) is

$$
\phi(k)=\int d p p \hat{K}(k, p, E) \phi(p),
$$

but now with ${ }^{3,4}$

$$
\begin{aligned}
\hat{\boldsymbol{K}}(k, p, E)= & 2 \Theta(\Lambda-p) \Theta(\Lambda-k) t\left(E-\frac{3}{4} p^{2}\right) \\
& \times\left[\left(k^{2}+p^{2}-E\right)^{2}-k^{2} p^{2}\right]^{-1 / 2},
\end{aligned}
$$

where $t\left(E-\frac{3}{4} p^{2}\right)$ is the appropriate two-body $s$-wave $t$ matrix. The effective range parametrization of the twobody $t$ matrix in this case is given by

$$
\begin{aligned}
t(\epsilon) & =2\left[\ln (-\epsilon)-\ln \gamma^{2}\right]^{-1} \\
& =2\left[\ln \left(-\epsilon / \gamma^{2}\right)\right]^{-1} .
\end{aligned}
$$

This two-body $t$ matrix has a bound-state pole at $\epsilon=-\gamma^{2}$, and the appropriate Efimov limit of zero twobody binding is achieved when $\gamma \rightarrow 0$. The form (11) for the two-body $t$ matrix is appropriate for our analysis though a one-term attractive separable potential produces a zero-energy bound state only in the limit when the strength of the potential goes to zero. However, a two-term separable potential with an attractive and a repulsive part can produce a zero-energy bound state with a nonzero potential. Even in this latter case the low-energy parametrization of the two-particle $t$ matrix can be written in the form of Eq. (11). As in the case of three-dimensional problems we can study the Efimov and Thomas effects through a study of $\operatorname{Tr} \widehat{K}^{n}$. For $n=1, \operatorname{Tr} \hat{K}$ is given by

$$
\begin{aligned}
\operatorname{Tr} \hat{K}=4 \int_{0}^{\Lambda} p d p\left[\ln \left[\frac{\frac{3}{4} p^{2}-E}{\gamma^{2}}\right]\right]^{-1} \\
\times\left[\left(2 p^{2}-E\right)^{2}-p^{4}\right]^{-1 / 2}
\end{aligned}
$$

This form of $\operatorname{Tr} \hat{K}$ is appropriate for studying both the Efimov and the Thomas effects. The Efimov effect is related to low-momentum divergence of this integral, whereas the Thomas effect is related to its highmomentum divergence. In the case of the Efimov effect, the two-body binding energy $\gamma^{2}$ is zero and the pole of the two-body $t$ matrix in Eq. (12) coincides with the lower limit of the integral as one also takes $E=0$ in Eq. (12) as in Sec. II. In the case of the Thomas effect, the divergence of the integral (12) at the lower limit is excluded. For our purpose it is convenient to rewrite Eq. (12) as

$$
\begin{aligned}
\operatorname{Tr} \hat{K}=\lim _{\epsilon \rightarrow 0} 4 \int_{\epsilon}^{\Lambda} p d p\left[\ln \left(\frac{\frac{3}{4} p^{2}-E}{\gamma^{2}}\right)\right]^{-1} \\
\times\left[\left(2 p^{2}-E\right)^{2}-p^{4}\right]^{-1 / 2},
\end{aligned}
$$

or

$$
\begin{aligned}
\operatorname{Tr} \hat{K}=\lim _{\epsilon \rightarrow 0} 4 \int_{\epsilon / \gamma}^{\Lambda / \gamma} y d y\left[\ln \left[\frac{3}{4} y^{2}+\beta^{2} / \gamma^{2}\right]\right]^{-1} \\
\times\left[\left(2 y^{2}+\beta^{2} / \gamma^{2}\right)^{2}-y^{4}\right]^{-1 / 2},
\end{aligned}
$$

where $\beta^{2}=-E$. Equation (13) should be compared with Eq. (8); in both cases both Efimov and Thomas effects are related to the large $y$ divergence. There is one difference, however. In Eq. (13) while studying Efimov effect $(\gamma \rightarrow 0$, $\beta=0$ ) the lower limit of the integral cannot and should not extended to zero, because for $\beta=0$ the integral in Eq. (13) diverges at the lower limit as $y \rightarrow 0$; whereas in Eq. (8) the integral is perfectly finite at the lower limit. This is why the limit $\epsilon \rightarrow 0$ has been introduced in Eq. (13). On the other hand, while studying the Thomas effect we set $E=-\beta^{2} \neq 0$ and $\beta^{2}>\gamma^{2}$ so that the denominators of Eq. (13) never have a zero. It is easy to see that for $\beta=0$, $\operatorname{Tr} \hat{K}$ diverges as $\ln [\ln (\Lambda / \gamma)]$ while either $\gamma \rightarrow 0$ (Efimov limit) or $\Lambda \rightarrow \infty$ (Thomas limit); for $\beta \neq 0, \operatorname{Tr} \hat{K}$ diverges as $\ln [\ln (\Lambda)]$ as $\Lambda \rightarrow \infty$ (Thomas limit) and $\gamma$ is kept finite; but $\operatorname{Tr} \widehat{R}$ does not diverge for $\beta \neq 0, \gamma \rightarrow 0$, and $\Lambda$ finite. This behavior is identical to the behavior of $\operatorname{Tr} \hat{K}$ in three dimensions. But in order for the Efimov or the Thomas effect to exist $\operatorname{Tr} \widehat{\mathcal{R}}^{n}$ must diverge in a similar fashion for any $n$. For our purpose we consider $\operatorname{Tr} \widehat{K}^{2}$ for $E=0$, which can be easily written as

$$
\begin{aligned}
\operatorname{Tr} \hat{K}^{2} & =16 \int_{0}^{p=\Lambda} d p^{2} \int_{0}^{k=\Lambda} d k^{2}\left[\left(k^{2}+p^{2}\right)^{2}-k^{2} p^{2}\right]^{-1}\left[\ln \left(\frac{3 p^{2}}{4 \gamma^{2}}\right) \ln \left(\frac{3 k^{2}}{4 \gamma^{2}}\right)\right]^{-1} \\
& =\int_{0}^{(3 / 4)\left(\Lambda^{2} / \gamma^{2}\right)} d x \int_{0}^{(3 / 4)\left(\Lambda^{2} / \gamma^{2}\right)} d y\left[\left(x^{2}+y^{2}+x y\right)(\ln x \ln y)\right]^{-1}
\end{aligned}
$$


when written in the form of Eq. (14), the divergence of the trace coming only from the upper limit is of interest because the two-body binding energy $\gamma^{2}$ has been scaled from the integrand and put in the upper limit together with $\Lambda$. The most divergent part of the integral (14) at the upper limit can be extracted after making the following transformation of variables: $x=r \cos \theta, y=r \sin \theta$. Then we have

$$
\begin{aligned}
& \operatorname{Tr} \hat{K}^{2}=16 \int^{(3 / 4)\left(\Lambda^{2} / \gamma^{2}\right)} \frac{d r}{r} \\
& \times \int_{0}^{\pi / 2} d \theta(1+\cos \theta \sin \theta)^{-1} \\
& \quad \times[\ln (r \cos \theta) \ln (r \sin \theta)]^{-1} .
\end{aligned}
$$

At the upper limit of $r$ integration, presumably at $r \rightarrow \infty$, the $\theta$ integration is well behaved and any divergence in this limit should come from the upper limit of the following integral:

$$
\operatorname{Tr} \widehat{K}^{2} \sim \int^{(3 / 4)\left(\Lambda^{2} / \gamma^{2}\right)} \frac{d r}{r}(\ln r)^{-2}
$$

which at the upper limit yields

$$
\operatorname{Tr} \hat{K}^{2} \sim\left[\ln \left(\frac{3}{4} \Lambda^{2} / \gamma^{2}\right)\right]^{-1},
$$

which is perfectly finite as $\Lambda \rightarrow \infty$ or $\gamma \rightarrow 0$. This proves the absence of both Efimov and Thomas effects in two dimensions. The absence of the Efimov-Thomas divergence of the trace of the kernel in two dimensions means that the integration limit in Eq. (9) can conveniently be pushed to infinity without the need of introducing form factors of two-particle interaction. Hence, in Eq. (9) the large momentum behavior is essentially given by Eq. (10) even in the case of realistic potentials because, unlike in the case of three dimensions, at large momentum the kernel tends to zero very rapidly and gives convergent integrals at large momentum without any need of realistic form factors of two-particle interactions. In other words, Eq. (9) will be insensitive to the large momentum behavior of these form factors, or to the short-range behavior of the two-particle interactions. Assuming that in various three-particle calculations one uses different shortrange two-particle interactions, which produce identical two-particle on-shell results, then the three particle observables in two dimensions, unlike in three dimensions, will be reasonably model independent.

Though the absence of the Efimov effect in two dimensions has been conjectured before (using different models) by Bruch and Tjon ${ }^{4}$ (separable potential model), Lim and Mourone $^{13}$ (hyperspherical harmonic approach), and Lim and Shimer ${ }^{13}$ (Born-Oppenheimer approach), the present discussion is distinct from these works in being completely model independent and in being a unique unified discussion of the Efimov and Thomas effects and the model independence of three-particle observables in two dimensions.

The model independence of three-particle observables in two dimensions was implicit in the work of Tjon. ${ }^{3} \mathrm{He}$ found a small sensitivity of three-particle binding energy in two dimensions on two-particle interaction model employed when he held fixed the two-particle binding energy. We shall see that the above sensitivity disappears if one holds both the two-particle scattering length and binding energy fixed. Then the three-particle binding energy in two dimensions becomes essentially model independent.

\section{NUMERICAL RESULTS}

In order to demonstrate our claim of model independence in two dimensions of three-particle observables, we have performed three-particle calculations with the following separable potential ${ }^{3}$

$$
\left\langle\mathbf{p}|V| \mathbf{p}^{\prime}\right\rangle=-\lambda g(p) g\left(p^{\prime}\right),
$$

with

$$
g(p)=\left(\sigma^{2}+p^{2}\right)^{-m} .
$$

The two-particle $t$ matrix at energy $E$ with this potential is given by

$$
\left\langle\mathbf{p}|t(E)| \mathbf{p}^{\prime}\right\rangle=-\lambda g(p) g\left(p^{\prime}\right)\left[1+2 \pi \lambda \int_{0}^{\infty} q d q g^{2}(q)\left(E+i \epsilon-q^{2}\right)^{-1}\right]^{-1} \text {. }
$$

In this case the fully on-shell $t$ matrix $\langle k|t(E)| k\rangle \equiv 2 \pi\left\langle\mathbf{k}|t(E)| \mathbf{k}^{\prime}\right\rangle, k^{2}=k^{\prime 2}=E$, is given by

$$
\langle k|t(E)| k\rangle=\left(-\frac{\left(\sigma^{2}+E\right)^{2 m}}{2 \pi \lambda}+\frac{1}{2} \ln \sigma^{2}-\frac{1}{2} \ln E-\frac{1}{2} \sum_{j=1}^{2 m} \frac{\left(\sigma^{2}+E\right)^{j}}{j \sigma^{2 j}}+\frac{i \pi}{2}\right)^{-1} \text {. }
$$

Using the effective-range expansion, this $t$ matrix is parametrized at low energies as

$$
\langle k|t(E)| k\rangle=\frac{2}{\pi}(-\cot \delta+i)^{-1},
$$

with $^{14}$

$$
\cot \delta=a_{2}+\frac{1}{\pi} \ln E+b E+c E^{2}+\cdots .
$$

Here $\delta$ is the scattering phase shift ${ }^{14}$ and $a_{2}$ is the two- dimensional equivalent of the scattering length in three dimensions. It has been shown in Ref. 14 that there are several ways of defining the scattering length in this case in terms of $a_{2}$. However, for our purpose we shall call this dimensionless quantity $a_{2}$, the scattering length which is the energy-independent part of the twodimensional effective-range function $\cot \delta$. We also define a modified scattering length $\bar{a}_{2}$ by

$$
\cot \delta=\bar{a}_{2}+\frac{1}{\pi} \ln \left(\frac{E}{E_{2}}\right)+b^{\prime}\left(\frac{E}{E_{2}}\right)+\cdots
$$


so that

$$
\bar{a}_{2}=a_{2}+\frac{1}{\pi} \ln E_{2},
$$

where $E_{2}$ is the binding energy of the two-particle system. The modified scattering length $\bar{a}_{2}$ will be useful in our study of universality in the three-particle system in two dimensions. It is interesting to note that when the two-particle binding energy $E_{2}=1, \bar{a}_{2}$ reduces to $a_{2}$. In Eqs. (19) and (21) there is a parameter in the argument of the logarithm ${ }^{14}$ which is set equal to unity and takes care of dimensional consistency. Explicitly, Eqs. (17) and (19) lead to the following nonzero part of $\cot \delta$ in the limit $E \rightarrow 0$ :

$$
\begin{aligned}
\cot \delta & =\sigma^{4 m} / \pi^{2} \lambda+\frac{1}{\pi} \sum_{j=1}^{2 m-1} \frac{1}{j}+\frac{1}{\pi} \ln \frac{E}{\sigma^{2}} \\
& =\frac{\sigma^{4 m}}{\pi^{2} \lambda}+\frac{1}{\pi} \ln \frac{E_{2}}{\sigma^{2}}+\frac{1}{\pi} \sum_{j=1}^{2 m-1} \frac{1}{j}+\frac{1}{\pi} \ln \frac{E}{E_{2}} .
\end{aligned}
$$

It is obvious from Eqs. (22) and (23) that the apparent dimensional inconsistency of Eq. (19) does not really exist. From Eq. (23) we have explicitly

$$
\bar{a}_{2}=\frac{\sigma^{4 m}}{\pi^{2} \lambda}+\frac{1}{\pi} \ln \frac{E_{2}}{\sigma^{2}}+\frac{1}{\pi} \sum_{j=1}^{2 m-1} \frac{1}{j}
$$

and

$$
a_{2}=\frac{\sigma^{4 m}}{\pi^{2} \lambda}+\frac{1}{\pi} \ln \frac{1}{\sigma^{2}}+\frac{1}{\pi} \sum_{j=1}^{2 m-1} \frac{1}{j} .
$$

In order to demonstrate our claim of model indepen-

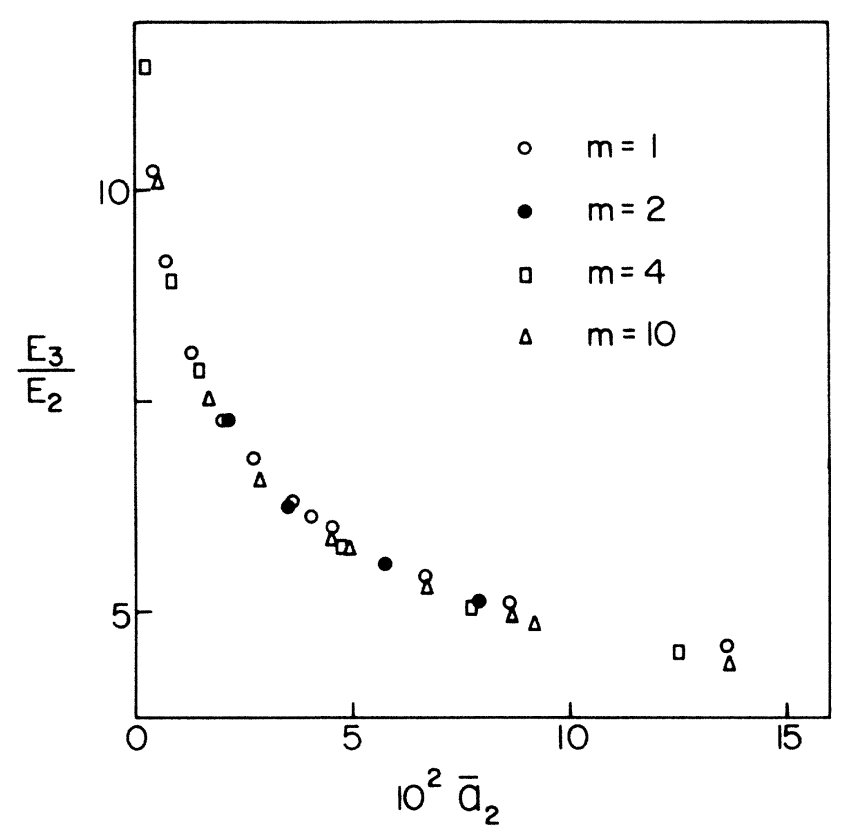

FIG. 1. The ratio of trimer and dimer binding energies $E_{3} / E_{2}$ as a function of $\bar{a}_{2}$ for the various separable potentials. Data of Tables I and II are plotted in this universal plot.

dence of three-particle observables in two dimensions, we calculate the binding energy $E_{3}$ of three identical bosons in two dimensions by solving the three-particle Faddeev equations in the momentum space.

The momentum-space Faddeev equations in the case of the separable two-particle interaction (15) can be written at energy $E=-s$ as

$$
\begin{aligned}
\phi(\mathbf{k}) & =2 \int d^{2} p\left(s^{2}+p^{2}+k^{2}+\mathbf{p} \cdot \mathbf{k}\right)^{-1} \frac{\lambda g\left(\left|\mathbf{p}+\frac{\mathbf{k}}{2}\right| \mid g\left(\left|\mathbf{k}+\frac{\mathbf{p}}{2}\right|\right)\right.}{\left(1+\lambda \int_{0}^{\infty} d^{2} q g^{2}(q)\left(-s-\frac{3}{4} p^{2}\right)^{-1}\right]} \phi(\mathbf{p}) \\
& \equiv \int d^{2} p K(\mathbf{k}, \mathbf{p}) \phi(\mathbf{p}) .
\end{aligned}
$$

The $s$-wave projection of Eq. (26) is

$$
\phi_{0}(k)=\int_{0}^{\infty} p d p K_{0}(k, p) \phi_{0}(p)
$$

with

$$
K_{0}(k, p)=\int_{0}^{2 \pi} d \theta K(\mathbf{k}, \mathbf{p}),
$$

where $\theta$ is the angle between vectors $\mathbf{k}$ and $\mathbf{p}$. Equation (27) is then solved by standard numerical techniques for the binding energy $E_{3}$ of the $s$-wave three-boson system interacting via the separable two-particle interaction (15). The two-boson binding energy $E_{2}$ is obtained by directly equating the energy denominator of Eq. (17) to zero. In Fig. 1 we plot the ratio of trimer to dimer binding energies $E_{3} / E_{2}$ versus $\bar{a}_{2}$. We varied the parameter $m$ of Eq. (16) from 1 to 10 and varied $\lambda$ of Eq. (15), setting $\sigma=1$ so as to generate some of the points of the $E_{3} / E_{2}$ versus $\bar{a}_{2}$ plot of Fig. 1. These points are exhibited in Table I and compared with the calculation of Ref. 3. Each value of $m$ of Eq. (16) generates one type of form factor. We find that the points for all $m$ 's lie on the same universal $E_{3} / E_{2}$ versus $\bar{a}_{2}$ plot.

The universal $E_{3} / E_{2}$ versus $\bar{a}_{2}$ plot is essentially the $E_{3}$ versus $a_{2}$ plot where $E_{2}=1$. This is because when $E_{2}=1, E_{3} / E_{2}$ reduces to $E_{3}$ and $\bar{a}_{2}$ reduces to $a_{2}$. This means that when $E_{2}=1, E_{3}$ is uniquely determined by $a_{2}$ and this unique value can be read off from Fig. 1. In order to verify this universality directly, we performed some calculations by varying $\sigma$ and $\lambda$ so as to maintain $E_{2}=1$. These results are shown in Table II. The results of Table II are also plotted in Fig. 1. It is easy to verify that the potentials of Table II use values of $\sigma$ which are very different from the values of $\sigma$ used in Table I. Yet the results of Tables I and II fall on the same universal 
TABLE I. The dimer binding energy $E_{2}$, scattering length $\bar{a}_{2}$, and the trimer to dimer binding energy ratio $E_{3} / E_{2}$ for various potentials characterized by $\sigma(=1), m$, and $\lambda$. The $E_{3} / E_{2}$ values are compared with the calculation of Tjon (Ref. 3) when available.

\begin{tabular}{|c|c|c|c|c|c|}
\hline$m$ & $\lambda$ & $E_{2}$ & $\bar{a}_{2}$ & $E_{3} / E_{2}$ & $\begin{array}{c}E_{3} / E_{2} \\
\text { (Reference 3) }\end{array}$ \\
\hline \multirow{10}{*}{1} & 0.0602 & 0.0019 & 0.0069 & 9.21 & \multirow[t]{2}{*}{9.05} \\
\hline & 0.0698 & 0.0040 & 0.0129 & 8.08 & \\
\hline & 0.0799 & 0.0073 & 0.0208 & 7.24 & \multirow[t]{2}{*}{7.26} \\
\hline & 0.0863 & 0.0100 & 0.0266 & 6.83 & \\
\hline & 0.0961 & 0.0150 & 0.0352 & 6.34 & \multirow{6}{*}{6.13} \\
\hline & 0.1000 & 0.0173 & 0.0401 & 6.18 & \\
\hline & 0.1043 & 0.0200 & 0.0449 & 6.01 & \\
\hline & 0.1242 & 0.0350 & 0.0673 & 5.44 & \\
\hline & 0.1404 & 0.0500 & 0.0863 & 5.12 & \\
\hline & 0.1838 & 0.1000 & 0.1366 & 4.58 & \\
\hline \multirow{4}{*}{2} & 0.0801 & 0.0032 & 0.0199 & 7.30 & \multirow{4}{*}{$\begin{array}{l}7.25 \\
6.26 \\
5.56 \\
5.12\end{array}$} \\
\hline & 0.0998 & 0.0074 & 0.0350 & 6.23 & \\
\hline & 0.1200 & 0.0135 & 0.0575 & 5.57 & \\
\hline & 0.1400 & 0.0211 & 0.0791 & 5.14 & \\
\hline \multirow{5}{*}{4} & 0.0481 & 0.0001 & 0.0019 & 11.53 & \\
\hline & 0.0633 & 0.0005 & 0.0075 & 8.97 & \\
\hline & 0.0731 & 0.0010 & 0.0133 & 7.91 & \\
\hline & 0.1116 & 0.0050 & 0.0468 & 5.82 & \\
\hline & 0.1861 & 0.0200 & 0.1245 & 4.55 & \\
\hline \multirow{5}{*}{10} & 0.0561 & 0.0001 & 0.0042 & 10.05 & \\
\hline & 0.0776 & 0.0005 & 0.0160 & 7.55 & \\
\hline & 0.0923 & 0.0010 & 0.0278 & 6.61 & \\
\hline & 0.1131 & 0.0020 & 0.0472 & 5.78 & \\
\hline & 0.1562 & 0.0050 & 0.0914 & 4.99 & \\
\hline
\end{tabular}

curve in Fig. 1, which is essentially the plot of $E_{3}$ versus $a_{2}$ for $E_{2}=1$. As all the potentials lie on the same universal curve of Fig. 1 we conclude that when $E_{2}$ is maintained constant $\left(E_{2}=1\right)$, the three-particle energy $E_{3}$ is a function of $a_{2}$ essentially independent of potential.

The same result is expected to hold for another $E_{2}$ as there is nothing special about $E_{2}=1$. It is interesting to comment at this stage that though the results of Tables I and II produce very different values of $E_{2}$, they are near the universal Efimov limit of small two-particle binding in that $E_{2} \ll<\sigma^{2}$ and the three-particle binding $E_{3}$ is reasonably model independent. Of course, there is a width of the $E_{3} / E_{2}$ versus $\bar{a}_{2}$ plot of Fig. 1 which varies from 0 (for $\bar{a}_{2}=0$ ) to $5 \%$ (for $\bar{a}_{2}=0.15$ ) as one moves away from the Efimov limit $\left(E_{2}=\bar{a}_{2}=0\right)$. It is well known ${ }^{8}$ that in a three-dimensional three-nucleon system as $E_{2}$ and $a_{2}$ are held fixed, $E_{3}$ varies widely, generating triton binding which may range from 6 to $12 \mathrm{MeV}$. From the preceding discussion of the Efimov and Thomas effects, such a large variation of $E_{3}$ in three dimensions is

TABLE II. The trimer binding energy $E_{3}$ for fixed $E_{2}(=1)$ and $a_{2}$ for various potentials characterized by $\sigma, m(=1$ and 10$)$, and $\lambda$. The calculated $E_{3}$ is roughly constant.

\begin{tabular}{rccccc}
\hline \hline$m$ & $\sigma$ & $\lambda / \sigma^{4 m}$ & $a_{2}$ & $E_{3}$ & $\left(\frac{E_{3}(m=1)}{E_{3}(m=10)}\right)$ \\
\hline 1 & 10.000 & 0.08630 & 0.02655 & 6.832 & 1.023 \\
10 & 32.539 & 0.09033 & 0.02655 & 6.681 & \\
1 & 7.071 & 0.10460 & 0.04485 & 6.014 & 1.027 \\
10 & 23.122 & 0.110728 & 0.04485 & 5.857 & 1.030 \\
1 & 5.345 & 0.12420 & 0.06727 & 5.442 & 1.032 \\
10 & 17.562 & 0.132902 & 0.06727 & 5.283 & \\
1 & 4.472 & 0.14043 & 0.08626 & 5.120 & 1.035 \\
10 & 14.75 & 0.15119 & 0.08626 & 4.961 & 4.582 \\
1 & 3.1623 & 0.18383 & 0.1366 & 4.425 & \\
10 & 10.509 & 0.20074 & 0.1366 &
\end{tabular}


expected. Though we are limited in this paper to a class of separable potentials of Eq. (15) (which results in, at best, a variation $E_{3}$ of the order of $<5 \%$ ) in view of the arguments presented in Sec. III, the present conclusion of model independence of three-particle observables in two dimensions is by no means limited to the example studied in this section and is expected to be true in general.

The present conclusion of model independence is expected to be true not only in the case of the three-particle binding energy $E_{3}$, but also for other low-energy threeparticle observables such as the scattering length $a_{3}$ for scattering of one particle from the bound state of other two. We calculated $a_{3}$ for our model potentials and found that this was indeed the case. Finally, we studied the correlation among $E_{3}$ and $a_{3}$ calculated with model potentials which produce the same $E_{2}$ and $a_{2}$. As expected from our study, the correlation between $E_{3}$ and $a_{3}$ is reduced essentially to a point if $E_{2}$ and $a_{2}$ are held fixed. In other words, if $E_{2}$ and $a_{2}$ are held fixed, the threeparticle system in two dimensions essentially produces the same $E_{3}$ and $a_{3}$ near the Efimov limit. In the case of the three-particle system in three dimensions, the on-shell properties of the two-particle system $E_{2}$ and $a_{2}$ are not sufficient to determine $E_{3}$ and $a_{3}$ uniquely. This leads to the well-known linear correlation ${ }^{8}$ between the calculated $E_{3}$ and $a_{3}$ in the three-nucleon system known as the Phillips plot.

\section{SUMMARY}

In this paper we have presented a unified discussion of the Efimov and Thomas effects and the model dependence of three-particle observables. In particular we have shown that both Efimov and Thomas effects are consequences of the same singularity structure of the kernel of the scattering integral equation satisfied by the three-particle system. In the case of the two-dimensional three-particle system, this singularity structure does not allow for the occurrence of Efimov and Thomas effects and suppresses the contributions of the large momentum parts of the two-particle $t$ matrix in the three-particle integral equation so that the three-particle observables are insensitive to large momentum parts or short-range behavior of the two-particle interaction. This makes the three-particle observables in two dimensions reasonably model independent. The three-particle system in three dimensions allows for the occurrence of Efimov and Thomas effects and consequently the three-particle observables in three dimension are very sensitive to shortrange (off-shell) behavior of two-particle interactions.

\section{ACKNOWLEDGMENTS}

This work is supported in part by the Conselho Nacional de Desenvolvimento-Científico e Tecnológico and Financiadora de Estudos e Projectos of Brazil.
1J. G. Dash and M. Schick, in The Physics of Liquid and Solid Helium, edited by K. H. Benneman and J. B. Ketterson (Wiley, New York, 1978); J. G. Dash, Phys. Rep. 38C, 177 (1978).

${ }^{2}$ A. Lagendijk, Phys. Rev. B 25, 2054 (1982); E. V. L. Mello, J. J. Rehr, and O. E. Vilches, ibid. 28, 3859 (1983).

${ }^{3}$ J. A. Tjon, Phys. Rev. A 21, 1334 (1980).

${ }^{4}$ M. L. Cramer, L. W. Bruch, and F. Cabral, J. Chem. Phys. 67, 1442 (1977); L. W. Bruch and J. A. Tjon, Phys. Rev. A 19, 425 (1979); F. Cabral and L. W. Bruch, J. Chem. Phys. 70, 4669 (1979).

${ }^{5}$ R. L. Siddon and M. Schick, Phys. Rev. A 9, 907 (1974); W. G. Gibson, Mol. Phys. 49, 103 (1983); Phys. Rev. A 36, 564 (1987).

${ }^{6}$ V. Efimov, Phys. Lett. 33B, 563 (1970); Nucl. Phys. A362, 45 (1981).
${ }^{7}$ L. H. Thomas, Phys. Rev. 47, 903 (1935).

${ }^{8}$ D. D. Brayshaw, Phys. Rev. Lett. 32, 382 (1974); A. C. Phillips, Nucl. Phys. A107, 209 (1968).

${ }^{9}$ L. Tomio, A. Delfino, and S. K. Adhikari, Phys. Rev. C 35, 441 (1987).

${ }^{10}$ S. K. Adhikari, Phys. Rev. C 30, 31 (1984); B. A. Girard and M. G. Fuda, ibid. 19, 583 (1979).

${ }^{11}$ R. D. Amado and J. V. Noble, Phys. Lett. 35B, 25 (1971); Phys. Rev. D 5, 1992 (1972).

${ }^{12}$ A. C. Fonseca, E. F. Redish, and P. E. Shanley, Nucl. Phys. A320, 273 (1979).

${ }^{13}$ T. K. Lim and P. A. Mourone, Phys. Rev. B 22, 1467 (1980); T. K. Lim and B. Shimer, Z. Phys. A 297, 185 (1980).

${ }^{14}$ S. K. Adhikari, W. G. Gibson, and T. K. Lim, J. Chem. Phys. 85, 5580 (1986); S. K. Adhikari, Am. J. Phys. 54, 362 (1986). 